quale la piccola borghesia lancia la sua protesta contro la società." Il borghese pirandelliano ha una coscienza etica della realtà. Perciò ragiona, giudica ed accusa disperatamente. Egli è costantemente propenso a scandagliare l'essenza della condizione umana, e in questa ricerca manifesta un comportamento risalente alla tradizione satirica e menippea (Il fu Mattia Pascal, Si gira).

Il critico indica tre aspetti importanti del romanzo pirandelliano. Il "racconto" che a volte attinge a fonti diverse della tradizione, cerca di rinnovarsi, sorreggendosi anche su intrecci contrastanti e complicati (Il fu Mattia Pascal), a volte rivela una "robusta tradizione epica" ( $I$ vecchi e $i$ giovani), a volte è rigoroso, didascalico ed intenso a concatenare anche elementi emblematici (Suo marito), mentre in Uno, nessuno e centomila il romanziere elimina la componente della trama, un segno importante di avanguardismo. Il secondo aspetto è quello del "discorso," l'insieme, cioè, dei procedimenti impiegati dall'autore per organizzare la fabula, quale la scelta della terza o della prima persona; il discorso rivolto dal narratore al lettore; o quello che il narratore traduce in soliloquio, o quello che a volte presuppone un destinatario. Il terzo aspetto è quello del "personaggio," che è per Pirandello l'elemento centrale del romanzo, specie quando lo costringe nella forma "moderna del raisonneur ingabbiato da una soffocante maschera." Il personaggio del romanzo pirandelliano, dal Fu Mattia Pascal a Uno, nessuno $e$ centomila, si fa sempre piú ricco, sempre piú maturo, sempre piú importante.

Lo studio felice di Gioviale è scritto in uno stile scorrevole e stringato. $\grave{E}$ corredato da tale dovizia di note da fame una vera e propria bibliografia, note che si divulgano in generose citazioni, spiegano dati e motivi storici, largiscono suggerimenti e interpretazioni. L'autore si avvale di numerose teorie narrative, da quelle dei formalisti russi a quelle degli strutturalisti francesi, e dei narratologi americani.

\title{
FRANCO ZANGRILLI
}

City University of New York

Anna Laura Lepschy. Narrativa e teatro fra due secoli. Verga, Invernizio, Svevo, Pirandello. Firenze: Leo S. Olschki, 1984. Pp. 248.

L'autrice in questa raccolta di dodici saggi, di cui nove giaè apparsi in riviste di italianistica, si serve di varie teorie critiche linguistiche per analizzare opere di Verga, Invemizio, Svevo e Pirandello. Come la Lepschy spiega nella Premessa, i saggi "si legano, per la loro occasione e per il loro svolgimento, a una lettura precisa e articolata di singoli testi, si tratti di cogliere certi aspetti nei rapporti fra narratore e narrativa (I, X), di identificare regolaritae strutturali latenti al di sotto di una apparente disorganizzazione (III) o nella costruzione ciell'intreccio (II, VII, XII), di specificare corrispondenze tematiche e strutturali fra certe opere di un autore (IV, XI) o di autori diversi (VIII, IX), di indagare 1'atteggiamento dello scrittore di fronte alle lingue e ai dialetti che conosce (V, VI)" (7).

Toma tutto a merito dell'autrice che le complicate teorie linguistiche di cui si serve nei suoi studi siano sempre chiaramente spiegate e che le sue analisi testuali servano ad una migliore comprensione dell'opera e dello scrittore, 
anziché diventare, come spesso succede, un'esemplificazione di questa o quella metodologia.

Nel primo saggio "Pirandellismo verghiano: "Di là del mare e il mondo dello scrittore" la Lepschy si avvale delle nozioni di ramificazione e di inclusione provenienti dalla linguistica chomskiana per chiarire la complessa struttura delle tre novelle "Nedda," Fantasticheria," e "Di là del mare" dove Verga include se stesso tra i personaggi descritti. L'autrice mette in rilievo il contrasto tra le due prime novelle, dove il Verga presenta se stesso sia come narratore narrante sia come narratore narrato, e la terza novella, dove il Verga separa il narratore dallo scrittore-personaggio. Tale contrasto, strutturale, secondo la Lepschy, "conferisce alle novelle un carattere de-costruttivo, come di autoanalisi metanarrative" (18).

L'autrice conclude che mentre il Verga nelle prime due novelle mette il mondo degli umili in secondo piano da usare come oggetto di interesse al mondo borghese e aristocratico a cui egli ambisce di appartenere, nella terza novella invece egli dà maggior importanza e permanenza all'atto dello scrivere e ai personaggi inventati dallo scrittore-personaggio che ai sentimenti di questi e della sua amante. Insomma in "Di là del mare" il Verga ci suggerisce pirandellianamente che qui è l'opera che "vive una vita piú reale di quella del suo autore" (20). Tramite l'analisi di una novella finora messa da parte dalla critica la Lepschy ci mostra dunque un interessante sviluppo della prospettiva del Verga verso se stesso in quanto narratore e verso il messaggio della sua opera.

Nel secondo studio "Aspetti della tecnica narrativa di Verga in Vita dei campi e Novelle rusticane: prolessi e analessi" l'autrice si rifâ sia all'uso genettiano di prolessi ed analessi sia alla distinzione tra fabula e intreccio dei formalisti russi per identificare in queste due raccolte di novelle la tecnica narrativa del Verga e in particolare il modo in cui lo scrittore ora anticipa al lettore gli avvenimenti della storia, ora spiega i precedenti di una situazione.

Ancora una volta tale analisi non è fine a se stessa ma porta alle interessanti conclusioni che in Vita dei campi Verga usa un maggior numero di prolessi perché "non pone in primo piano la storia, ma l'effetto che essa ha sui personaggi, e spesso ci informa in anticipo su quello che accadrà"; in Novelle rusticane invece usa un maggior numero di analessi perché "ci presenta una situazione o un atteggiamento" e vuole spiegarne le cause (36).

Le stesse abilità analitiche e sintetiche rilevate nei primi due saggi si ritrovano negli altri dieci, sia che l'autrice esplori l'elaborazione strutturale e le rispondenze tematiche del capitolo II dei Malavoglia (III), sia che paragoni la versione dialettale e quella italiana dello stesso romanzo di Carolina Invernizio (IV), sia che commenti l'atteggiamento di Svevo di fronte alla lingua italiana, ai dialetti e alle lingue straniere che conosce (V, VI), sia che esamini la funzione del tempo nella Coscienza di Zeno (VII), sia che paragoni La coscienza di Zeno col romanzo dello psicanalista Groddek Lo scrutatore d'anime (VIII) o Senilitd di Svevo con La noia di Moravia (IX).

Concludono la raccolta tre saggi su Pirandello. Nel primo la Lepschy analizza la figura dell'attore nelle opere pirandelliane in termini di contrasto, parallelo e fusione fra i personaggi che sono attori e quelli che non lo sono. L'autrice si serve della nozione di Hjelmslev di metalinguaggio per definire il metateatro di Pirandello in cui "abbiamo degli attori che interpretano attori che interpre- 
tano personaggi"(189) e alla fine osserva che "il fascino provato contemplando il meccanismo semiotico, attraverso il quale l'arte rispecchia il suo oggetto, si moltiplica e si intensifica quando il riflesso stesso si riflette nella commedia e nella narrativa"(203).

Nel secondo saggio la Lepschy esamina la struttura ciclica delle commedie di Pirandello, in cui alla fine si ritoma al punto di partenza, e mette in rapporto questa struttura colla psicologia dei personaggi pirandelliani che "quanto piú hanno vissuto una loro parte, tanto piué difficile trovano allontanarsene"(225).

Infine nel terzo saggio l'autrice esamina l'ambiguità delle situazioni e dei rapporti umani nella commedia Tutto per bene ed esplora i vari aspetti della negazione presenti nell'opera, usando le categorie freudiane di rigetto completo di una situazione spiacevole (Verwerfung), di disconoscimento di quello che di fatto si vede (Verleugnung) e di negazione di un contenuto rimosso, che in questo modo penetra nella coscienza, a patto di venire nagato (Verneinung).

In conclusione dunque il libro della Lepschy è un prezioso ausilio agli studiosi dell'ottocento e del Novecento per la varietà dei temi affrontati, per la serietà e l'impegno delle analisi compiute e per la novità delle prospettive offerte ai lettori.

GIULIANA SANGUINETTI KATZ University of Toronto 